\title{
Nucleotide Excision Repair, XPA-1, and Translesion Synthesis Complex, POLZ-1 and REV-1, are Critical for Interstrand Crosslink Repair in C. elegans Germ Cells
}

\author{
Sinae Oh ${ }^{1, \dagger}$, Woori Bae ${ }^{1,4, \dagger}$, Mohammad A. Alfhili², Myon Hee Lee ${ }^{3, *}$ \\ 'Department of Biochemistry, College of Life Science and Biotechnology, Yonsei \\ University, 03772, Seoul, South Korea. ${ }^{2}$ Chair of Medical and Molecular Genetics Research, \\ Department of Clinical Laboratory Sciences, College of Applied Medical Sciences, King \\ Saud University, Riyadh, 12372, Saudi Arabia. ${ }^{3}$ Department of Internal Medicine, \\ Hematology/Oncology Division, Brody School of Medicine at East Carolina University, \\ Greenville, NC 27834, United States. ${ }^{4}$ Current address: Department of Epigenetics and \\ Molecular Carcinogenesis, University of Texas MD Anderson Cancer Center, Houston, TX \\ 77030, United States. ${ }^{\dagger}$ These authors contributed equally to this work.
}

*Corresponding author: Myon Hee Lee, Ph.D

Email: leemy@ecu.edu; Tel: +1 (252) 744-3134 


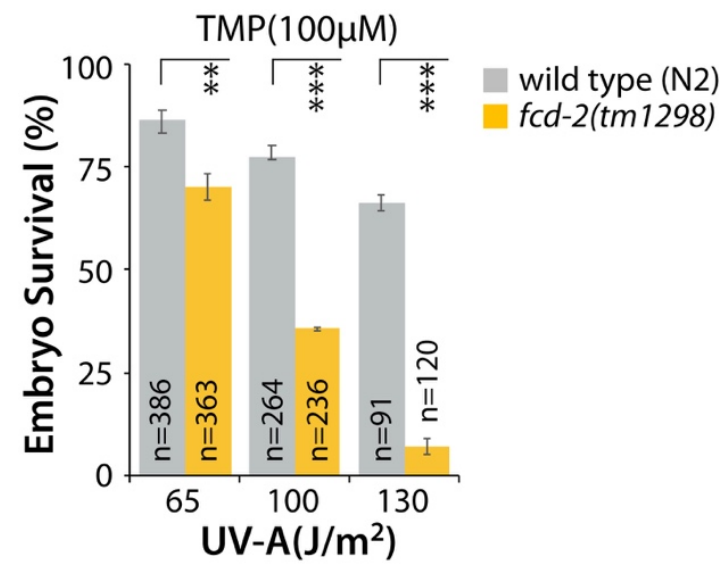

Figure S1. UV-A dose-dependent effect on C. elegans germ cells. L4 stage worms were treated with different UV-A doses in the presence of $100 \mu \mathrm{M}$ TMP. Level of germ cell damages by TMP/UV-A exposure was determined by scoring the survival rate of the fertilized embryos. $P$ values: ${ }^{*}, P<0.05 ;{ }^{* *}, P<0.01 ;{ }^{* *}, P<0.001$; ns, no statistical significance. See Table S2 for One-way ANOVA analysis.

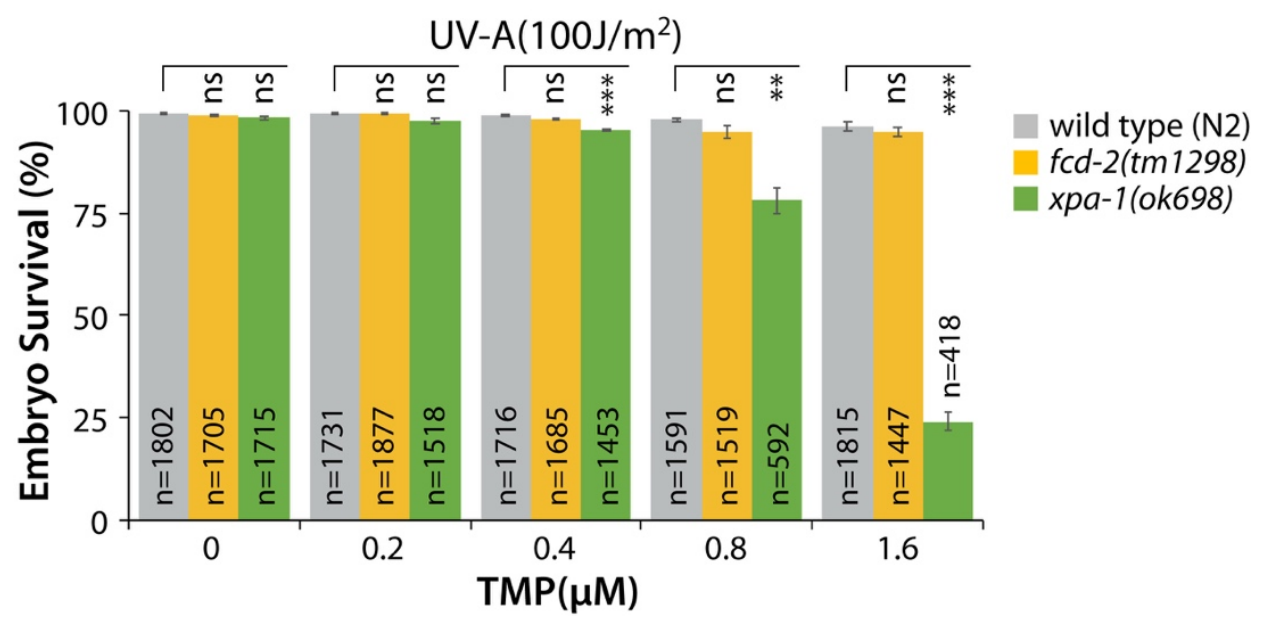

Figure S2. TMP dose-dependent effect on C. elegans germ cells. L4 stage worms were treated with different TMP doses in the presence of $100 \mathrm{~J} / \mathrm{m}^{2} \mathrm{UV}-\mathrm{A}$. Level of germ cell damages by TMP/UV-A exposure was determined by scoring the survival rate of the fertilized embryos. $P$ values: ${ }^{*}, P<0.05 ;{ }^{*}, P<0.01 ;{ }^{* *}, P<0.001 ;$ ns, no statistical significance. See Table $S 2$ for One-way ANOVA analysis. 


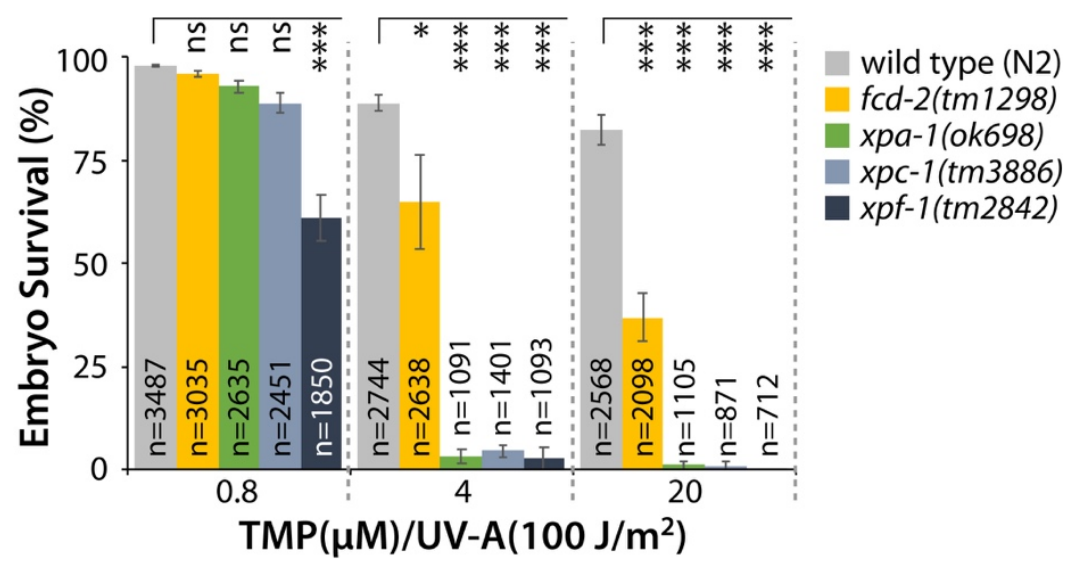

Figure S3. XP mutants were more sensitive to ICLs than fcd-2 mutants. $L 4$ stage worms were treated with TMP $(0.8$ and $1.6 \mu \mathrm{M})$ and exposed to UV-A $\left(100 \mathrm{~J} / \mathrm{m}^{2}\right)$ to score embryonic lethality. $P$ values: ${ }^{*}, P<0.05 ;{ }^{* *}, P<0.01$; ${ }^{* *}, P<0.001$; ns, no statistical significance. See Table S2 for One-way ANOVA analysis. 
Table S1. Strain information

\begin{tabular}{|c|c|c|}
\hline Strain & Mutant info. & Reference \\
\hline$x p a-1$ (ok698) & Null mutation & Denver et al. (2006) Genetics (PMID: 1569771) \\
\hline$x p f-1(t m 2842)$ & Null mutation & $\begin{array}{c}\text { Agostinho et al. (2013) PLoS Genetics } \\
\text { (PMID: 23901331) }\end{array}$ \\
\hline fcd-2(tm1298) & Null mutation & Adamo et al. (2010) Mol Cell (PMID: 20598602) \\
\hline polz-1(tm8927) & Nonsense mutation & None \\
\hline rev-1(tm8701) & Nonsense mutation & None \\
\hline
\end{tabular}

\section{The motif analysis of POLZ-1(tm8927) and REV-1(tm8701)}

Domain analysis was conducted by MOTIF search and NCBI Protein Blast (http://www.genome.jp/tools/motif/, http://blast.ncbi.nlm.nih.gov).

\section{A. Motif analysis of POLZ-1}

\section{polz-1(tm8927) mutation site 269 bp deletion (5790/5791 - 6059/6060)}

ATGAACTTTTGCATTCGAAATTTTATATGTGAGCACAGTCAAGAAATTCCGGGACCTATGGATACTGTATATAACTTTCGAAGGAATAAAA TCCCTGTTTTTCATATTTATGGAGTGACTGATACCTATCAAAAGGCATGTCTACATATTCATGGAGTTCTTCCATATCTTGTTCTTCGTGT TGGAAATAAAGTGACTCCATCTGTGTTGGCTGCGATGAGAGCAAAAGTTAATAAAGGAATTGAAAAGGAAATTGAGACGAGCACTGGAAAA ACGAACAAGTTCTCTGTGGATTACGTTTACAAAATGGAGAGCTTCAGTAGTCGCTCACTCTACGGATATCAAGATGAAGAAGAAGACTTTG TTCGAGTTTATTTTTCATCTCCATGGTATCTTAAAAAAGCTACGCATTCACTTGGAAAAGAAGTCATCGATAAACCACTTTTTCAACCATA CGAGGCTCATCTTCCTTTTCATCTCCAGTTTTTCATTGATAATTCCATATTTGGAATGGATAATATTTATCTGAAAAAAGTCAAATTTCGA ATGGATTCGAGGGATGAAGATGCAACGTTGATTTATAAAGATTTGTCAGTTGGCGATATCAGAAATAATGAGCTCTTATTGTCTCCATACG AGAAGAAGACAACTTGCCACGTGGAATGTGATGCATTGGTTACAGATATTCTGAATATGGAGATGCAGGCAGATAATGTTCACTCTTCCAA CCCTGGATTGGAATATCTTTGGAGAGAGGAGAAAGAGAGGTGTGATGCACTTGGAATTGAACTCAAGGATGTATTTAATTCATATGAACC CGAAAATCAACAATCTGTCCTCAAGAACGCGACATGTTACGAACCGCCCGAAAAATGGCTAAGAAATTCCGACAAGAAAGGTGTATGTCGG AACAGTTGGATGATCTGATGGTCACAAGAATTGCCGAATCTCAGCAGACAACGTCATCAGTGGGTGCATCGGACACGACAATATGGGAGCA GCCAGGCGAGGATCCTATGGAAGAGGACCGAATAGGCAGGGAGAAAACTCCTGAAGAACTGGACGCTGAACGTCAGAAAGAAGAAGATCGC GAAGAGGCAGCAGAAGATGATGATAATGATCCAAAAAATCAGGAAGCAGAAATGACGATGTTTGCTGATAGTCAAAAACCTGTTGAAAGAG ATGAAGCTATGGATGTAGATGAAGAAGTCGAAGATGATGAAGAATTGAATGACAAGGGAACGGGGGAAGTGATTGAAATCAGTTCGGATGG AGATGATGATCAAGACATTGTTCAATGGATCACAACGAAAAGCGCGTATGATCAAAATTTCATCAGCATGAACGAATTCCTATTTGATGCC AAGAAAATTGAAAATCAACGGCAGTTCTCTCGGGAAATGACTGCTTGTTCTGTGATGACTGATGAGACAGTAGTTGCTGCAGCTGAACATT CACAACCTGTCGAACAGACACGTCTGCTCTCACAAACTTCAATTGGTGAACCTTCTACTTCTGAGAAAAGTGTTGAATCGGACATTGTTGG ATTATGTGTGGCAAGTTTAGAGCTTCTAGTCGACACAAAAATGCCAATGCCAGACGCAGCTTCCAGTGAAATTGTGTCAGTGTCATTAGCA ATTTACAACGATGTATGTCGTTGTTCATCTCCGAAGCTTCATGTTCTTCTCACAACCTTACCTGTTGGACCGGCATATTCAGATCGAAGAG TTGTTCACATGGAGACTGAATTGGAAATGTTGGAAGAAGTCGCTAAATGGATTGTACAATACGATGTTGATGTTATGATCGGATACGAGAC CATTCGTTTATCATGGGGATTCTTTTTCCGTAGAATCAAACTCCTTGGATCTAGAATCTCAATGGATCGAGCGTTGATTGATGCCTACGA GATCATATCGAAGTAGACGATCAAGAAATCACAGTTGCTCCACCAAAAGGAAGACTTCTAGTTTCAGTATGGAAAGTTGTTCGTAGTGATC TAGCACTTCGGAATTATGATCTGGGAAGTGCTGTGGCAAATGTGTTACGAAAAAAAATTCCGATGTTGGATAATGCTGCGTTGATGAGAAG AATCAAAGGAGAAAGGTCTGCCATAAGAAACGATGTACATTTGCATCTTCTAAAACTGTCTAGCCTTAACATATCTCTTCTAACCGAAATG AATTGGTTCCTGAAAAACGCTGAAATGGCACGAGTATATGGAATTCAATTTCACGAAGTTTGGACTCGAGGATCCCAGTTACGAGTGGAAT CCATGCTTCTCCGTTTGGCACATCGTATGAATTTTGTGGCTCCGTCAATTACCCATTTGCAGAGAAACATGATGGGCTCTCCGGAGCAGCT TCAACTTATTCTGGAACCCCAATCAAAAGTCTATTTTGATCCGGTTATTGTTCTAGATTTCCAATCATTGTATCCATCAATGGTTATTGCA TACAACTACTGCTACTCAACTATTTTGGGAAAAATTGGAAATTTAGTACAAATGAATGATGAGTCCAGGAATCGAGAAGAGATTGTACTTC GAGCAATCAAGTACCATCCTAGTAAAGATGATATTGTGAAGTTGGTGGCCTATAAAGAAGTCTGTGCATCTCCACTTGCATCAATGTTTGT GAAAAAGTCAAAACGGGAAGGTGTGATGCCTTTGTTGCTCAGAGAGATTCTCGCCGCTCGAATAATGGTGAAAAGTGCGATGAAACGGACG AAAAACAAGAAGCTGAAACGAATTCTAGATGCTCGACAACTGGCTCTGAAACTTGTTGCAAATGTGTCATATGGATATACAGCAGCGAATT GGAGTGGACGAATGCCTTGTGCAGAATTAGCCGATGCCATCCTCGGAAAAGGTCGTGAGACTCTGGAGAGATCAATTGAAATGGTTCAACG AGGTGAT (269 bp deletion)

GTGTCCAATTGTAGCAGAAGTTCTCGAAAAGTCACTTGGGTTGATTTTCTCTCAAAATTGGAAAACCTTTATCACTTATCTGAATACAGTA GTACTAAATCTACCACAAGAGAATTTCTCAAAATTCGTATTCTGCAAAGAGTATAGAGGTGATTACTCGGCACGAGCGATGGTACCACAAA AGAAAATTGCAGAAGCTCGAATCAGAAAGTGTCCATCCCACATAACACTACGTGGGGAGCGTGTACCTTACGTTATCATTGATGGTGTCAC CGGATCTACAGTATACTCTTGTGTACGGTCAATAGATCAGTTTGCTCGTAATCCGGAATACAAGATCAACACTTTCTACTATTTGAATGCT CATATTCTTGCTGCTCTGCGAAGAGTCACTGATTTGATTCCAATGAAGATTGACTTCTTACCATTTGCTGCGGAGCAGTGTTTTGTGTCTG ACTGCTCGAGAATCGGAAAGACTCCATGGTGCATTGAATGCGAGCAAAATCCAGAAGAACTCGCTCTAGCTCTAGTTCAATTGGGACGAGA GAGTCGTGCTAGATCCCAAATTATACAATATTGCACAAGCTGTCAATCTTCCAGTCAACGTCTTGGCGAAGATCAAGTAGTCGATTGTGCC AACTTTACATGTCTACTACGGCAGACTATGTCAATTATGGATCGTTCAAGAACCGCGTCAGTTCTGACTGCACATAAGATGTTCTAA

Wild type POLZ-1 amino acid sequence and motifs

MNFCIRNF I CEHSQE I PGPMDTVYNFRRNK I PVFHI YGVT DTYQKACLHI HGVL PYLVLRVGNKVTPSVLAAMRAKVNKGIEKE I E TSTGKTNKFSVDYVYKMESFSSRS LYGYQDEEEDFVRVYFSSPWYLKKATHSLGKEVI DKPLFQPYEAHLPFHLQFF I DNS I FGMD

NIYLKKVKFRMDSRDEDATL IYKDLSVGD I RNNELLLSPYEKKTTCHVECDALVTDI LNMEMQADNVHSSNPGLEYLWREEKERCD ALGIELKDVFNSYEPRKST ICPQERDMLRTARKMAKKFRQERCMSEQLDDLMVTRIAESQQTTSSVGASDTT IWEQPGEDPMEEDR IGREKTPEELDAERQKEEDREEAAEDDDNDPKNQEAEMTMFADSQKPVERDEAMDVDEEVEDDEELNDKGTGEVIEISS DGDDDQD IVQWITTKSAYDQNF ISMNEFLFDAKK IENQRQF SREMTACSVMTDETVVAAAEHSQPVEQTRLLSQTSIGEPSTSEKSVESDIVG LCVASLELLVDTKMPMPDAASSE IVSVSLA I YNDVCRCSS PKLHVLLTTLPVGPAYSDRRVVHMETELEMLEEVAKWIVQYDVDVM 
IGYET IRLSWGFF FRR I KLLGSR I SMDRAL I DAYEDH IEVDDQE ITVAPPKGRLLVSVWKVVRS DLALRNYDLGSAVANVLRKKIP MLDNAALMRR IKGERSA IRNDVHLHLLKLSS LN I SLLTEMNWF LKNAEMARVYG IQFHEVWTRGSQLRVESMLLRLAHRMNFVAPS ITHLQRNMMGSPEQLQL ILEPQSKVYF DPVIVLDFQS LYPSMV IAYNYCYSTI LGKI GNLVQMNDESRNREE IVLGAIKYHPSKDD IVKLVAYKEVCAS PLASMFVKKSKREGVMPLLLREI LAAR IMVKSAMKRTKNKKLKRI LDARQLALKLVANVSYGYTAANWSGRMP CAELADA I LGKGRETLERS I EMVQRGDYGGAEV I YGDTDSMFVLVRGASVEEAFE IGRRIVDDVTNSNPDPVVLKLEKVYKGCVLE TKKRYAGWMYEHENDEGSLDAKG IETVRRDTCP IVAEVLEKSLGLIFS QNWKTF I TY LNTVVLNL PQENFSKFVFCKEYRGDYSAR AMVPQKKIAEARIRKCPSH ITLRGERVPYVI IDGVTGSTVYSCVRS I DOFARNPEYKINTFYYLNAH I LAALRRVTDLI PMK I DFL PFAAEQCFVSD SRTASVLTAHKMF

POLZ-1(tm8927) amino acid sequence and motifs - nonsense mutation

MNFCIRNF ICEHSQEI PGPMDTVYNFRRNKI PVFHIYGV

TSTGKTNKESVDYVYKMESFSSRSLYGYQDEEEDFVRVYFSSPWYLKKATHSLGKEVIDKPLFQPYEAHLPEHLQFFIDNS I FGMD
NIYLKKVKERMDSRDEDATLIYKDLSVGDIRNNELLLSPYEKKTTCHVECDALVTDI LNMEMQADNVHSSNPGLEYLWREEKERCD ALGIELKDVFNSYEPRKSTICPQERDMLRTARKMAKKFRQERCMSEQLDDLMVTRIAESQQTTSSVGASDTTIWEQPGEDPMEEDR IGREKTPEELDAERQKEEDREEAAEDDDNDPKNQEAEMTMFADSQKPVERDEAMDVDEEVEDDEELNDKGTGEVIEISSDGDDDQD IVQWITTKSAYDQNF I SMNEFLFDAKKIENQRQF SREMTACSVMTDETVVAAAEHSQPVEQTRLLSQTSIGEPSTSEKSVESDIVG LCVASLELLVDTKMPMP MLDNAALMRR IKGERSA IRNDVHLHLLKLS S LN I SLLTEMNWFLKNAEMARVYGIQFHEVWTRGSQLRVESMLLRLAHRMNFVAPS ITHLQRNMMGSPEQLQL ILEPQSKVYFDPVIVLDFQSLYPSMVIAYNYCYSTI LGKI GNLVQMNDESRNREE IVLGAIKYHPSKDD IVKLVAYKEVCAS PLASMFVKKSKREGVMP LLLREI LAAR IMVKSAMKRTKNKKLKRI LDARQLALKLVANVSYGYTAANWS GRMP CAELADAI LGKGRETLERS IEMVQRGDVSNCSRSSRKVTWVDFLSKLENLYHLSEYSSTKSTTREFLKIRILQRV (STOP)

*Additional amino acids are underlined

\begin{tabular}{|l|l|l|l|}
\hline Pfam ID & Description & Position & i-Evalue \\
\hline PF03104 & DNA polymerase family B, exonuclease & $40-184$ & $5.2 \mathrm{e}-10$ \\
\cline { 3 - 4 } DNA_pol_B_exo1 & domain & $532-675$ & $2.4 \mathrm{e}-06$ \\
\hline PF00136 DNA_pol_B & DNA polymerase family B & $744-1195$ & $1.9 \mathrm{e}-92$ \\
\hline PF14260 Zf-C4pol & C4-type zinc-finger of DNA polymerase delta & $1216-1279$ & 0.37 \\
\hline
\end{tabular}

\section{B. Motif analysis of REV-1}

\section{rev-1(tm8701) mutation site \\ 236 bp deletion +2 bp insertion \\ $(11170 / 11171-(A C)-11406 / 114087)$}

ATGCATTCTCCTGACCCATTCACCGATCATCCAGATTCAGATGATTCTTTTGAAAAGTTTGTTCCGACTCCTAATGTGAATCGACGACGAA GTGATGCTATAATTGTTTCAGATGAGGGTTCATTCGACGGAATAACAGGATATTCCCAAGTAACAGTGATCGAAAATTGTCCGAATACTAC TGTACTAGAAGATGAAAATAAACGCGCAAATCCTGATGATACAATAACTCGAATGCTAGAATCCAGTGACGAAGAGGATGAAACTGAAAAT AATTCTGATAATGATGCACTTGCTGATCTTTTAAAATCGTTCAGAACGGAAGATCAGAAAAGAAAAGAAGTAGACATTGAACAGGCAGCAA ATATCAGTATAGACATATTTGGTGATTTAATTGAAGAAGTTGACTCAGAACATGAAGCTGCTGAATATCCGGATAGAGAAGGTGGAAATAG CATGGATAAGCAAGGAACAATGCTACCGGTTGAAGTTCCTATTCAAAATAATATCAAACTTATACCAAGAAAGCGTCGTCTAGAACCAAAA TCATATGATTGTGAACCTTCAACATCCGCTGCACATGGCCCAGTTGGTGTTGTTTCATCAGATAGTGGCGAGTACTGGAATCAACAGAAAA CCATCACCATACATGGACAAAATTTCGAAATAAATAATTTTAATGACTATATGCGGATGAAGATCACTAAACTTAACCATCAGGTGAATCA TGGATATGCGAAACCACTAGAGAATGTGTCTAGAATAATGGAAGGATTCTCGGTATTTGTGAATGGTTATACAGATCCACCAGCACTTGTT ATCAGAGATTTGATGATTTCTCACGGAGGTGAATATCACTGTTATTATCAACATGGAATTACTTCATACACGATAGCTTCCTCAATTGCTA CAGCAAAAATAAATCGAATTCGAGAAAATGAAATTTTCATTAAAGCTGATTGGATTACGGAATCAATAGCTGCTGGAAAACCACTCGATTA TCGAGATTTTTTGATATACGAGAAAGGTTCGGTGGAAAAGGGACAAATGCAACAGTTTCTAACAATATCAACTAGAAACACCGGTGGTAAC AATGAAACAACTTCATCGAATCAGTTCTCTGATGCCCGGAATCCAAACTTTATTCGAGATTACTATGCCAGAAGTCGTCTTCATTTGATTT CTACTCTCGCGCAGGATATGAAAGATTTTGTGGCAAATTTGAAACTGGAAGGAAAGCTAACGGAAAAGTGTTTTGAAGAGAAAGAACTCAT CGATATGAAGTCTCTTAGCAATGAAATTTCTCGGGAAAGTACAGTATTCCACGTGGATCTAGACTGTTTCTTCGTCTCAGTTGCTGTCAGA AATCGAATTGATTTGAAACACAAAGAAGTTGCCATCACTCATAGCAAGGGAACTATTTCGAATAGCATGAGCGAGGTGGCTTCTTGCAGTT ATGCAGCTCGTGATTGTGGAGTCAAAAATGGTATGCTCGTGAGAGATGCTCTACAAAAGTGTCCACAATTAACTCTATTACCATATCAATT TGAGGATTATGTGCAAGTTAGTCGGAAAATTTATGAAATTCTGGCTAGCTACACATTGGAGGTTCGTGCAGTCTCCTGCGATGAAATGTAT ATAAACATGAGCAGTTTTTGTGAAAAATACGAAATCAACGACCCAACAATTCTCGCTGAACACATCCGTAAAGTGATACGAGAGAAGACTC AATGCCCAGCCAGTGTTGGAATAGGATCTACGAGCTTGTTAGCAAGACTTGCAACAAGACATGCTAAACCAGATGGAGTTTTTTGGGTTAA TGCACATAAGAAAAATGAATTCATTTCCGAGGAAAAAGTTAAAGATCTACCAGGATTTGGATACGAAATGATGAATAGATTAACTTCATTC TTTGGTGATATTACAAAATGCCGTGAACTACAATTAAAAACTGAACGGGAACTTGTACCTGTATTCGGACCAAAGTTGGCTTCAAAAATAT TACGACAATGTCGTGGTATTGAAGAAGATCCAGATGATTTTTGGGCTACTCATGTTCGAAAATCAGTATCATGTGATATCAATTATGGTAT TCGTTTCACGAAG (236 bp deletion + AC insertion)

TTCCAACTACACGCGGAGAGTCTCTCACATCAGAAGCTATGAAATTATATGCTAAAGTTTCTCCGAAAGTAGAAGATCTTCGTGGAGTTGG AGTTACCTGTGGAAAGTTGAAAAGTAAATTGAAAAAAGATGCGGCAACGGCAGTGCAAGAGATGTTTGGGAAGACCTCTAGAGTTGGGAAT ATGGCGAGAACTGACGAACAATTGAATATTATTCCGCGAAACGAAGATGAACTAGATAAAGAACCTGTGATAATTCCAGCTGTGACTGAAG ATCTGGAAGTGCAAAGCATACCTCGTCAAATTAATTTTAGAGTGGCAAATGACATAGATATTCCTGAAATTGTAAAATCCACACTTCTCAA TGGACGATCTGACAACAACCTCGAATTGGAAGATTTGGAAGATGATGGATTAGTTGAAAATCGTCTGGCCGAATTGGAATGTATTATTCAA AATGAGCCAACCAAGGAGAGTGTTACGAACTTACAGGAAATGCTTTCAACGTTGCTAAACTATGGAAAACTTTCCGCATTCGAGACTGTAT TCAGAAAGTTTGAAGAGATTTCGATGGATTCTAGTAGATGCGACACTGCTTGGTTTTCAGTATTCCATTTGATGGTACCATTCATCGAAGA AGAATCAAAAGATCTGCTTGAATTCCCATAGCTACCACTCGAAATTTGGAGCTATGATTGCCAGCAACACTGATAACACATCAATTAGT TCACCAGAGCCATTTGTTCCAAAAGAAGTATTTGTAATTGATTCCACTAAACATACCTGA 
Wild type REV-1 amino acid sequence and motifs

MHSPDPFTDHPDSDDSFEKFVPTPNVNRRRSDAI IVSDEGSFDGITGYSQVTVIENCPNTTVLEDENKRANPDDTITRMLESSDEEDETEN NSDNDALADLLKSFRTEDQKRKEVDIEQAANIS I DIFGDLIEEVDSEHEAAEYPDREGGNSMDKQGTMLPVEVPIQNNIKLIPRKRRLEPK SYDCEPSTSAAHGPVGVVSSDSGEYWNQQKT IT I HGQNEE INNFNDYMRMKITKLNHQVNHGYAKPLENVSRIMEGFSVFVNGYTDPPALV IRDLMI SHGGEYHCYYQHGITSYTIASS IATAK INR IRENEI F I KADWITES IAAGKPLDYRDFLIYEKGSVEKGQMQQFLT ISTRNTGGN NETTSSNQFSDARNPNF IRDYYARSRLHLISTLAQDMKDFVANLKLEGKLTEKCFEEKELI DMKSLSNEISRESTVFHVDLDCFFVSVAVR NRIDLKHKEVAITHSKGT ISNSMSEVASCSYAARDCGVKNGMLVRDALQKCPQLTLLPYQFEDYVQVSRKIYEILASYTLEVRAVSCDEMY INMSSFCEKYEINDPT I LAEH IRKVIREKTQCPASVGIGSTSLLARLATRHAKPDGVFWVNAHKKNEF ISEEKVKDLPGFGYEMMNRLTSF FGDITKCRELQLKTERELVPVFGPKLASKILRQCRGIEEDPDDFWATH

GSITLKLMVRSANAPIQTSKFMGHGICDTFTKTCNLNVPTTRGESLTSEAMKLYAKVSPKVEDLRGVGVTCGKLKSKLKKDAATAVQEMFG KTSRVGNMARTDEQLNI I PRNEDELDKEPVI I PAVTEDLEVQS I PRQ INFRVANDI D I PEIVKSTLLNGRSDNNLELEDLEDDGLVENRLA ELECI IQNEPTKESVTNLQEMLSTLLNYGKLSAFETVFRKFEE ISMDSSRCDTAWFSVFHLMVPFIEEESKDLLEFPIATTRNLGAMIASN TDNTSISSPEPEVPKEVEVIDSTKHT

\section{REV-1(tm8701) amino acid sequence and motifs - nonsense mutation}

MHSPDPFTDHPDSDDSFEKFVPTPNVNRRRSDA I IVSDEGSFDGITGYSQVTVIENCPNTTVLEDENKRANPDDTITRMLESSDEEDETEN NSDNDALADLLKSFRTEDQKRKEVDIEQAANIS I I FGDLIEEVDSEHEAAEYPDREGGNSMDKQGTMLPVEVPIQNNIKLIPRKRRLEPK SYDCEPSTSAAHGPVGVVSSDSGEYWNQQKTITIHGQNFE INNENDYMRMKITKLNHQVNHGYAKPLENVSRIMEGFSVFVNGYTDPPALV IRDLMI SHGGEYHCYYQHGITSYT IASS IATAKINR IRENE I F IKADWITES IAAGKP LDYRDFLIYEKGSVEKGQMQQFLT I STRNTGGN NETTSSNQFSDARNPNF IRDYYARSRLHLISTLAQDMKDFVANLKLEGKLTEKCFEEKELIDMKSLSNEISRESTVFHVDLDCFFVSVAVR NRIDLKHKEVAITHSKGT ISNSMSEVASCSYAARDCGVKNGMLVRDALQKCPQLTLLPYQFEDYVQVSRKIYEILASYTLEVRAVSCDEMY INMSSFCEKYE INDPTI LAEHIRKVIREKTQCPASVGIGSTSLLARLATRHAKPDGVFWVNAHKKNEFISEEKVKDLPGFGYEMMNRLTSF FGDITKCRELQLKTERELVPVFGPKLASKILRQCRGIEEDPDDFWAT

*Additional amino acids are underlined

\begin{tabular}{|l|l|l|l|}
\hline Pfam ID & Description & Position & i-Evalue \\
\hline PF16589 BRCT_2 & BRCT domain, a BRCA1 C-terminus domain & $257-337$ & 0.0019 \\
\hline PF00817 IMS & impB/musB/samB family & $443-595$ & $2.5 \mathrm{e}-39$ \\
\hline PF11799 IMS_C & impB/musB/samB family C-terminal domain & $685-804$ & $1.1 \mathrm{e}-11$ \\
\hline
\end{tabular}


Table S2. One-way ANOVA analysis.

Figure 2B (0 $\mu \mathrm{M}$ TMP)

\begin{tabular}{|lcc|}
\hline Tukey's multiple comparisons test & Significance & P Value \\
\hline Wild type (N2) vs. fcd-2(tm1298) & $\mathrm{ns}$ & 0.9225 \\
Wild type (N2) vs. xpa-1(ok698) & $\mathrm{ns}$ & 0.9273 \\
Wild type (N2) vs. xpf-1(tm2842) & $* * *$ & $<0.0001$ \\
fcd-2(tm1298) vs. xpa-1(ok698) & $\mathrm{ns}$ & 0.6285 \\
fcd-2(tm1298) vs. xpf-1(tm2842) & $* * *$ & $<0.0001$ \\
xpa-1(ok698) vs. xpf-1(tm2842) & $* * *$ & $<0.0001$ \\
\hline
\end{tabular}

Figure 2B (0.8 $\mu \mathrm{M}$ TMP)

\begin{tabular}{|lcc|}
\hline Tukey's multiple comparisons test & Significance & P Value \\
\hline Wild type (N2) vs. fcd-2(tm1298) & $\mathrm{ns}$ & 0.9878 \\
Wild type (N2) vs. xpa-1(ok698) & $\mathrm{ns}$ & 0.1658 \\
Wild type (N2) vs. xpf-1(tm2842) & $* * *$ & 0.0001 \\
fcd-2(tm 1298) vs. xpa-1(ok698) & $\mathrm{ns}$ & 0.2546 \\
fcd-2(tm1298) vs. xpf-1(tm2842) & $* * *$ & 0.0002 \\
xpa-1(ok698) vs. xpf-1(tm2842) & $* *$ & 0.001 \\
\hline
\end{tabular}

Figure 2B (4 $\mu \mathrm{M}$ TMP)

\begin{tabular}{|lcc|}
\hline Tukey's multiple comparisons test & Significance & P Value \\
\hline Wild type (N2) vs. fcd-2(tm1298) & $\mathrm{ns}$ & 0.0639 \\
Wild type (N2) vs. xpa-1(ok698) & $* * *$ & $<0.0001$ \\
Wild type (N2) vs. xpf-1(tm2842) & $* * *$ & $<0.0001$ \\
fcd-2(tm1298) vs. xpa-1(ok698) & $* * *$ & $<0.0001$ \\
fcd-2(tm1298) vs. xpf-1(tm2842) & $* * *$ & $<0.0001$ \\
xpa-1(ok698) vs. xpf-1(tm2842) & ns & 0.7757 \\
\hline
\end{tabular}

Figure 2B (20 $\mu \mathrm{M}$ TMP)

\begin{tabular}{|lcc|}
\hline Tukey's multiple comparisons test & Significance & P Value \\
\hline Wild type (N2) vs. fcd-2(tm1298) & $*$ & 0.0147 \\
Wild type (N2) vs. xpa-1(ok698) & $* * *$ & $<0.0001$ \\
Wild type (N2) vs. xpf-1(tm2842) & $* * *$ & $<0.0001$ \\
fcd-2(tm1298) vs. xpa-1(ok698) & $* *$ & 0.0011 \\
fcd-2(tm1298) vs. xpf-1(tm2842) & $* *$ & 0.0011 \\
xpa-1(ok698) vs. xpf-1(tm2842) & ns & $>0.9999$ \\
\hline
\end{tabular}

Figure 2C (0 $\mu \mathrm{M}$ TMP)

\begin{tabular}{|lcc|}
\hline Tukey's multiple comparisons test & Significance & P Value \\
\hline Wild type (N2) vs. fcd-2(tm1298) & $\mathrm{ns}$ & 0.9916 \\
Wild type (N2) vs. xpa-1(ok698) & $\mathrm{ns}$ & 0.913
\end{tabular}




\begin{tabular}{|lcc|} 
Wild type (N2) vs. polz-1(tm8927) & $\mathrm{ns}$ & $>0.9999$ \\
Wild type (N2) vs. rev-1(tm8701) & $\mathrm{ns}$ & 0.9047 \\
fcd-2(tm 1298) vs. xpa-1(ok698) & $\mathrm{ns}$ & 0.9934 \\
fcd-2(tm 1298) vs. polz-1(tm8927) & $\mathrm{ns}$ & 0.9967 \\
fcd-2(tm 1298) vs. rev-1(tm8701) & $\mathrm{ns}$ & 0.9918 \\
xpa-1(ok698) vs. polz-1(tm8927) & $\mathrm{ns}$ & 0.9414 \\
xpa-1(ok698) vs. rev-1(tm8701) & $\mathrm{ns}$ & $>0.9999$ \\
polz-1(tm8927) vs. rev-1(tm8701) & $\mathrm{ns}$ & 0.9348 \\
\hline
\end{tabular}

Figure 2C (0.8 $\mu \mathrm{M}$ TMP)

\begin{tabular}{|lcc|}
\hline Tukey's multiple comparisons test & Significance & P Value \\
\hline Wild type (N2) vs. fcd-2(tm1298) & $\mathrm{ns}$ & 0.9275 \\
Wild type (N2) vs. xpa-1(ok698) & $* * *$ & $<0.0001$ \\
Wild type (N2) vs. polz-1(tm8927) & $* *$ & 0.001 \\
Wild type (N2) vs. rev-1(tm8701) & $\mathrm{ns}$ & 0.6737 \\
fcd-2(tm1298) vs. xpa-1(ok698) & $* * *$ & $<0.0001$ \\
fcd-2(tm 1298) vs. polz-1(tm8927) & $* *$ & 0.003 \\
fcd-2(tm 1298) vs. rev-1(tm8701) & $\mathrm{ns}$ & 0.9793 \\
xpa-1(ok698) vs. polz-1(tm8927) & $* * *$ & 0.0004 \\
xpa-1(ok698) vs. rev-1(tm8701) & $* * *$ & $<0.0001$ \\
polz-1(tm8927) vs. rev-1(tm8701) & $* *$ & 0.0066 \\
\hline
\end{tabular}

Figure $2 \mathrm{C}$ ( $1.6 \mu \mathrm{M}$ TMP)

\begin{tabular}{|lcc|}
\hline Tukey's multiple comparisons test & Significance & P Value \\
\hline Wild type (N2) vs. fcd-2(tm1298) & $\mathrm{ns}$ & 0.989 \\
Wild type (N2) vs. xpa-1(ok698) & $* * *$ & $<0.0001$ \\
Wild type (N2) vs. polz-1(tm8927) & $* * *$ & $<0.0001$ \\
Wild type (N2) vs. rev-1(tm8701) & $* * *$ & $<0.0001$ \\
fcd-2(tm 1298) vs. xpa-1(ok698) & $* * *$ & $<0.0001$ \\
fcd-2(tm 1298) vs. polz-1(tm8927) & $* * *$ & $<0.0001$ \\
fcd-2(tm 1298) vs. rev-1(tm8701) & $* * *$ & $<0.0001$ \\
xpa-1(ok698) vs. polz-1(tm8927) & $\mathrm{ns}$ & 0.5537 \\
xpa-1(ok698) vs. rev-1(tm8701) & $* * *$ & $<0.0001$ \\
polz-1(tm8927) vs. rev-1(tm8701) & $* * *$ & $<0.0001$ \\
\hline
\end{tabular}

Figure 2D (0 $\mu \mathrm{M}$ TMP)

\begin{tabular}{|lcc|}
\hline Tukey's multiple comparisons test & Significance & P Value \\
\hline Wild type (N2) vs. fcd-2(tm1298) & $\mathrm{ns}$ & $>0.9999$ \\
Wild type (N2) vs. xpa-1(RNAi) & $\mathrm{ns}$ & $>0.9999$ \\
Wild type (N2) vs. polz-1(tm8927) & $\mathrm{ns}$ & 0.9421 \\
Wild type (N2) vs. rev-1(tm8701) & $\mathrm{ns}$ & 0.7214 \\
Wild type (N2) vs. polz-1(tm8927);xpa-1(RNAi) & $\mathrm{ns}$ & $>0.9999$ \\
Wild type (N2) vs. rev-1(tm8701);xpa-1(RNAi) & $\mathrm{ns}$ & $>0.9999$
\end{tabular}


fcd-2(tm 1298) vs. xpa-1 (RNAi)

ns

0.997

fcd-2(tm 1298) vs. polz-1(tm8927)

ns

0.9878

fcd-2(tm 1298) vs. rev-1(tm8701)

0.8533

fcd-2(tm 1298) vs. polz-1(tm8927); xpa-1(RNAi)

ns

$>0.9999$

fcd-2(tm 1298) vs. rev-1(tm8701); xpa-1(RNAi)

ns

$>0.9999$

xpa-1(RNAi) vs. polz-1(tm8927)

0.8583

xpa-1(RNAi) vs. rev-1(tm8701)

ns

0.5896

xpa-1 (RNAi) vs. polz-1(tm8927); xpa-1(RNAi)

$>0.9999$

xpa-1(RNAi) vs. rev-1(tm8701); xpa-1 (RNAi)

0.9989

polz-1 (tm8927) vs. rev-1(tm8701)

0.9967

polz-1 (tm8927) vs. polz-1(tm8927); xpa-1(RNAi)

0.9441

polz-1(tm8927) vs. rev-1(tm8701); xpa-1(RNAi)

0.9784

rev-1(tm8701) vs. polz-1(tm8927); xpa-1(RNAi)

0.7255

rev-1(tm8701) vs. rev-1(tm8701); xpa-1(RNAi)

0.8153

polz-1(tm8927); xpa-1 (RNAi) vs. rev-1(tm8701); xpa-1(RNAi)

$>0.9999$

Figure 2D (0.8 $\mu \mathrm{M}$ TMP)

\begin{tabular}{|lcc|}
\hline Tukey's multiple comparisons test & Significance & P Value \\
\hline Wild type (N2) vs. fcd-2(tm1298) & $\mathrm{ns}$ & $>0.9999$ \\
Wild type (N2) vs. xpa-1(RNAi) & $\mathrm{ns}$ & $>0.9999$ \\
Wild type (N2) vs. polz-1(tm8927) & $\mathrm{ns}$ & $>0.9999$ \\
Wild type (N2) vs. rev-1(tm8701) & $\mathrm{ns}$ & 0.9995 \\
Wild type (N2) vs. polz-1(tm8927);xpa-1(RNAi) & $\mathrm{ns}$ & 0.3598 \\
Wild type (N2) vs. rev-1(tm8701);xpa-1(RNAi) & $*$ & 0.036 \\
fcd-2(tm1298) vs. xpa-1(RNAi) & $\mathrm{ns}$ & $>0.9999$ \\
fcd-2(tm1298) vs. polz-1(tm8927) & $\mathrm{ns}$ & $>0.9999$ \\
fcd-2(tm1298) vs. rev-1(tm8701) & $\mathrm{ns}$ & $>0.9999$ \\
fcd-2(tm1298) vs. polz-1(tm8927);xpa-1(RNAi) & $\mathrm{ns}$ & 0.4069 \\
fcd-2(tm1298) vs. rev-1(tm8701);xpa-1(RNAi) & $*$ & 0.0411 \\
xpa-1(RNAi) vs. polz-1(tm8927) & $\mathrm{ns}$ & $>0.9999$ \\
xpa-1(RNAi) vs. rev-1(tm8701) & $\mathrm{ns}$ & $>0.9999$ \\
xpa-1(RNAi) vs. polz-1(tm8927);xpa-1(RNAi) & $\mathrm{ns}$ & 0.4772 \\
xpa-1(RNAi) vs. rev-1(tm8701);xpa-1(RNAi) & $*$ & 0.0493 \\
polz-1(tm8927) vs. rev-1(tm8701) & $\mathrm{ns}$ & $>0.9999$ \\
polz-1(tm8927) vs. polz-1(tm8927);xpa-1(RNAi) & $\mathrm{ns}$ & 0.4574 \\
polz-1(tm8927) vs. rev-1(tm8701);xpa-1(RNAi) & $*$ & 0.0469 \\
rev-1(tm8701) vs. polz-1(tm8927);xpa-1(RNAi) & $\mathrm{ns}$ & 0.537 \\
rev-1(tm8701) vs. rev-1(tm8701);xpa-1(RNAi) & $\mathrm{ns}$ & 0.057 \\
polz-1(tm8927);xpa-1(RNAi) vs. rev-1(tm8701);xpa-1(RNAi) & $\mathrm{ns}$ & 0.5048 \\
\hline
\end{tabular}

Figure 2D (1.6 $\mu \mathrm{M}$ TMP)

Tukey's multiple comparisons test

Significance P Value 
Wild type (N2) vs. fcd-2(tm1298)

Wild type (N2) vs. xpa-1 (RNAi)

Wild type (N2) vs. polz-1(tm8927)

Wild type (N2) vs. rev-1 (tm8701)

Wild type (N2) vs. polz-1(tm8927); xpa-1(RNAi)

Wild type (N2) vs. rev-1(tm8701); xpa-1(RNAi)

fcd-2(tm 1298) vs. polz-1(tm8927); xpa-1(RNAi)

fcd-2(tm1298) vs. rev-1(tm8701); xpa-1(RNAi)

xpa-1(RNAi) vs. polz-1(tm8927)

хра-1(RNAi) vs. rev-1(tm8701)

xpa-1(RNAi) vs. polz-1(tm8927); xpa-1(RNAi)

хра-1(RNAi) vs. rev-1(tm8701); хра-1 (RNAi)

polz-1 (tm8927) vs. rev-1(tm8701)

polz-1(tm8927) vs. polz-1(tm8927); xpa-1(RNAi)

$* * * \quad<0.0001$

$* * *$

$<0.0001$

$* *$

0.0017

*** $\quad 0.0001$

polz-1(tm8927) vs. rev-1(tm8701); xpa-1(RNAi)

$* * *$

$<0.0001$

rev-1(tm8701) vs. polz-1(tm8927); xpa-1(RNAi)

$<0.0001$

rev-1(tm8701) vs. rev-1(tm8701); хра-1 (RNAi)

$* * *$

$<0.0001$

polz-1(tm8927); xpa-1(RNAi) vs. rev-1(tm8701); xpa-1(RNAi)

$* * *$

Figure $3 \mathrm{C}(+\mathbf{0} \mathrm{h})$

\begin{tabular}{|lcc|}
\hline Tukey's multiple comparisons test & Significance & P Value \\
\hline Wild type (N2) vs. xpa-1(RNAi) & $\mathrm{ns}$ & 0.793 \\
Wild type (N2) vs. rev-1(tm8701) & $\mathrm{ns}$ & 0.9591 \\
Wild type (N2) vs. rev-1(tm8701); xpa-1(RNAi) & $\mathrm{ns}$ & 0.9349 \\
xpa-1(RNAi) vs. rev-1(tm8701) & $\mathrm{ns}$ & 0.5249 \\
xpa-1(RNAi) vs. rev-1(tm8701);xpa-1(RNAi) & $\mathrm{ns}$ & 0.4769 \\
rev-1(tm8701) vs. rev-1(tm8701);xpa-1(RNAi) & $\mathrm{ns}$ & 0.9997 \\
\hline
\end{tabular}

Figure $3 C(+6 h)$

\begin{tabular}{|lcc|}
\hline Tukey's multiple comparisons test & Significance & P Value \\
\hline Wild type (N2) vs. xpa-1(RNAi) & $\mathrm{ns}$ & $>0.9999$ \\
Wild type (N2) vs. rev-1(tm8701) & $\mathrm{ns}$ & 0.1915 \\
Wild type (N2) vs. rev-1(tm8701); xpa-1(RNAi) & $*$ & 0.0414 \\
xpa-1(RNAi) vs. rev-1(tm8701) & $\mathrm{ns}$ & 0.1816 \\
xpa-1(RNAi) vs. rev-1(tm8701);xpa-1(RNAi) & $*$ & 0.0392 \\
rev-1(tm8701) vs. rev-1(tm8701);xpa-1(RNAi) & $\mathrm{ns}$ & 0.7026 \\
\hline
\end{tabular}

Figure $3 \mathrm{C}(+12 \mathrm{~h})$

Tukey's multiple comparisons test $\quad$ Significance $P$ Value

Wild type (N2) vs. xpa-1 (RNAi)

0.0386 


\begin{tabular}{|lll|} 
Wild type (N2) vs. rev-1(tm8701) & $* * *$ & $<0.0001$ \\
Wild type (N2) vs. rev-1(tm8701); xpa-1(RNAi) & $* * *$ & 0.0001 \\
xpa-1(RNAi) vs. rev-1(tm8701) & $* *$ & 0.0021 \\
xpa-1(RNAi) vs. rev-1(tm8701); xpa-1(RNAi) & $* *$ & 0.0047 \\
rev-1(tm8701) vs. rev-1(tm8701);xpa-1(RNAi) & ns & 0.9057 \\
\hline
\end{tabular}

Figure $3 \mathrm{C}(+18 \mathrm{~h})$

\begin{tabular}{|lcc|}
\hline Tukey's multiple comparisons test & Significance & P Value \\
\hline Wild type (N2) vs. xpa-1(RNAi) & $\mathrm{ns}$ & $>0.9999$ \\
Wild type (N2) vs. rev-1(tm8701) & $* * *$ & $<0.0001$ \\
Wild type (N2) vs. rev-1(tm8701);xpa-1(RNAi) & $* * *$ & $<0.0001$ \\
xpa-1(RNAi) vs. rev-1(tm8701) & $* * *$ & $<0.0001$ \\
xpa-1(RNAi) vs. rev-1(tm8701);xpa-1(RNAi) & $* * *$ & $<0.0001$ \\
rev-1(tm8701) vs. rev-1(tm8701);xpa-1(RNAi) & $\mathrm{ns}$ & 0.0671 \\
\hline
\end{tabular}

Figure $3 C$ (+24 h)

\begin{tabular}{|lcc|}
\hline Tukey's multiple comparisons test & Significance & P Value \\
\hline Wild type (N2) vs. xpa-1(RNAi) & $\mathrm{ns}$ & 0.9996 \\
Wild type (N2) vs. rev-1(tm8701) & $\mathrm{ns}$ & 0.3989 \\
Wild type (N2) vs. rev-1(tm8701);xpa-1(RNAi) & $* * *$ & $<0.0001$ \\
xpa-1(RNAi) vs. rev-1(tm8701) & $\mathrm{ns}$ & 0.4468 \\
xpa-1(RNAi) vs. rev-1(tm8701); xpa-1(RNAi) & $* * *$ & $<0.0001$ \\
rev-1(tm8701) vs. rev-1(tm8701);xpa-1(RNAi) & $* * *$ & $<0.0001$ \\
\hline
\end{tabular}

Figure 4C (+12 h)

\begin{tabular}{|lcc|}
\hline Tukey's multiple comparisons test & Significance & P Value \\
\hline Wild type(N2) vs. xpa-1(RNAi) & $\mathrm{ns}$ & 0.8329 \\
Wild type(N2) vs. rev-1(tm8701) & $\mathrm{ns}$ & 0.9182 \\
Wild type(N2) vs. rev-1(tm8701); xpa-1(RNAi) & $\mathrm{ns}$ & 0.4203 \\
xpa-1(RNAi) vs. rev-1(tm8701) & $\mathrm{ns}$ & 0.9965 \\
xpa-1(RNAi) vs. rev-1(tm8701);xpa-1(RNAi) & $\mathrm{ns}$ & 0.8628 \\
rev-1(tm8701) vs. rev-1(tm8701);xpa-1(RNAi) & $\mathrm{ns}$ & 0.7611 \\
\hline
\end{tabular}

Figure 4C (+24 h)

\begin{tabular}{|lcc|}
\hline Tukey's multiple comparisons test & Significance & P Value \\
\hline Wild type(N2) vs. xpa-1(RNAi) & $\mathrm{ns}$ & 0.9998 \\
Wild type(N2) vs. rev-1(tm8701) & $* *$ & 0.0087 \\
Wild type(N2) vs. rev-1(tm8701);xpa-1(RNAi) & $*$ & 0.012 \\
xpa-1(RNAi) vs. rev-1(tm8701) & $* *$ & 0.0097 \\
xpa-1(RNAi) vs. rev-1(tm8701);xpa-1(RNAi) & $*$ & 0.0134 \\
rev-1(tm8701) vs. rev-1(tm8701);xpa-1(RNAi) & $\mathrm{ns}$ & 0.9945 \\
\hline
\end{tabular}


Figure $5(0.8 \mu \mathrm{M}$ TMP)

\begin{tabular}{|lcc|}
\hline Tukey's multiple comparisons test & Significance & P Value \\
\hline Wild type (N2) vs. fcd-2(tm1298) & $\mathrm{ns}$ & $>0.9999$ \\
Wild type (N2) vs. xpa-1(ok698) & $\mathrm{ns}$ & 0.4403 \\
Wild type (N2) vs. polz-1(tm8927) & $\mathrm{ns}$ & 0.9255 \\
Wild type (N2) vs. rev-1(tm8701) & $\mathrm{ns}$ & $>0.9999$ \\
Wild type (N2) vs. polz-1(tm8927); rev-1(tm8701) & $* * *$ & $<0.0001$ \\
fcd-2(tm1298) vs. xpa-1(ok698) & $\mathrm{ns}$ & 0.5577 \\
fcd-2(tm1298) vs. polz-1(tm8927) & $\mathrm{ns}$ & 0.9696 \\
fcd-2(tm1298) vs. rev-1(tm8701) & $\mathrm{ns}$ & $>0.9999$ \\
fcd-2(tm1298) vs. polz-1(tm8927); rev-1(tm8701) & $* * *$ & $<0.0001$ \\
xpa-1(ok698) vs. polz-1(tm8927) & $\mathrm{ns}$ & 0.9638 \\
xpa-1(ok698) vs. rev-1(tm8701) & $\mathrm{ns}$ & 0.5961 \\
xpa-1(ok698) vs. polz-1(tm8927);rev-1(tm8701) & $* * *$ & 0.0008 \\
polz-1(tm8927) vs. rev-1(tm8701) & $\mathrm{ns}$ & 0.9684 \\
polz-1(tm8927) vs. polz-1(tm8927); rev-1(tm8701) & $* * *$ & 0.0004 \\
rev-1(tm8701) vs. polz-1(tm8927);rev-1(tm8701) & $* * *$ & $<0.0001$ \\
\hline
\end{tabular}

Figure 5 (1.6 $\mu \mathrm{M}$ TMP)

\begin{tabular}{|lcc|}
\hline Tukey's multiple comparisons test & Significance & P Value \\
\hline Wild type (N2) vs. fcd-2(tm1298) & $\mathrm{ns}$ & 0.9893 \\
Wild type (N2) vs. xpa-1(ok698) & $* * *$ & $<0.0001$ \\
Wild type (N2) vs. polz-1(tm8927) & $* * *$ & $<0.0001$ \\
Wild type (N2) vs. rev-1(tm8701) & $* * *$ & $<0.0001$ \\
Wild type (N2) vs. polz-1(tm8927); rev-1(tm8701) & $* * *$ & $<0.0001$ \\
fcd-2(tm1298) vs. xpa-1(ok698) & $* * *$ & $<0.0001$ \\
fcd-2(tm1298) vs. polz-1(tm8927) & $* * *$ & $<0.0001$ \\
fcd-2(tm1298) vs. rev-1(tm8701) & $* * *$ & $<0.0001$ \\
fcd-2(tm1298) vs. polz-1(tm8927); rev-1(tm8701) & $* * *$ & $<0.0001$ \\
xpa-1(ok698) vs. polz-1(tm8927) & $\mathrm{ns}$ & 0.9735 \\
xpa-1(ok698) vs. rev-1(tm8701) & $* * *$ & $<0.0001$ \\
xpa-1(ok698) vs. polz-1(tm8927); rev-1(tm8701) & $* * *$ & $<0.0001$ \\
polz-1(tm8927) vs. rev-1(tm8701) & $* * *$ & $<0.0001$ \\
polz-1(tm8927) vs. polz-1(tm8927); rev-1(tm8701) & $* * *$ & $<0.0001$ \\
rev-1(tm8701) vs. polz-1(tm8927); rev-1(tm8701) & $* * *$ & $<0.0001$ \\
\hline
\end{tabular}

Figure $\mathrm{S} 1(65 \mathrm{~J} / \mathrm{m} 2)$

Tukey's multiple comparisons test Significance P Value

Wild type (N2) vs. fcd-2(tm1298) $\quad * * 0.0029$

Figure S1 (100 J/m2)

Tukey's multiple comparisons test Significance P Value 
Figure S1 (130 J/m2)

Tukey's multiple comparisons test Significance $\mathbf{P}$ Value

Wild type (N2) vs. fcd-2(tm1298) $\quad * * *<0.0001$

Figure S2 (0 $\mu \mathrm{M}$ TMP)

\begin{tabular}{|lcc|}
\hline Tukey's multiple comparisons test & Significance & P Value \\
\hline Wild type (N2) vs. fcd-2(tm1298) & $\mathrm{ns}$ & 0.5477 \\
Wild type (N2) vs. xpa-1(ok698) & $\mathrm{ns}$ & 0.106 \\
fcd-2(tm 1298) vs. xpa-1(ok698) & $\mathrm{ns}$ & 0.4127 \\
\hline
\end{tabular}

Figure S2 (0.2 $\mu \mathrm{M}$ TMP)

\begin{tabular}{|lcc|}
\hline Tukey's multiple comparisons test & Significance & P Value \\
\hline Wild type (N2) vs. fcd-2(tm1298) & $\mathrm{ns}$ & 0.9766 \\
Wild type (N2) vs. xpa-1(ok698) & $\mathrm{ns}$ & 0.0683 \\
fcd-2(tm 1298) vs. $x p a-1(o k 698)$ & $\mathrm{ns}$ & 0.0887 \\
\hline
\end{tabular}

Figure S2 (0.4 $\mu \mathrm{M}$ TMP)

\begin{tabular}{|lcc|}
\hline Tukey's multiple comparisons test & Significance & P Value \\
\hline Wild type (N2) vs. fcd-2(tm 1298) & $\mathrm{ns}$ & 0.0708 \\
Wild type (N2) vs. xpa-1(ok698) & $* * *$ & $<0.0001$ \\
fcd-2(tm 1298) vs. $x p a-1$ (ok698) & $* *$ & 0.0005 \\
\hline
\end{tabular}

Figure S2 (0.8 $\mu \mathrm{M}$ TMP)

\begin{tabular}{|lcc|}
\hline Tukey's multiple comparisons test & Significance & P Value \\
\hline Wild type (N2) vs. fcd-2(tm1298) & $\mathrm{ns}$ & 0.4883 \\
Wild type (N2) vs. xpa-1(ok698) & $* *$ & 0.0022 \\
fcd-2(tm1298) vs. xpa-1(ok698) & $* *$ & 0.0047 \\
\hline
\end{tabular}

Figure S2 (1.6 $\mu \mathrm{M}$ TMP)

\begin{tabular}{|lcc|}
\hline Tukey's multiple comparisons test & Significance & P Value \\
\hline Wild type (N2) vs. fcd-2(tm1298) & $\mathrm{ns}$ & 0.7508 \\
Wild type (N2) vs. xpa-1(ok698) & $* * *$ & $<0.0001$ \\
fcd-2(tm1298) vs. xpa-1(ok698) & $* * *$ & $<0.0001$ \\
\hline
\end{tabular}

Figure S3 (0.8 $\mu \mathrm{M}$ TMP)

\begin{tabular}{|lcc|}
\hline Tukey's multiple comparisons test & Significance & P Value \\
\hline Wild type (N2) vs. fcd-2(tm1298) & $\mathrm{ns}$ & 0.9985 \\
Wild type (N2) vs. xpa-1(ok698) & $\mathrm{ns}$ & 0.7146 \\
Wild type (N2) vs. xpc-1(tm3886) & $\mathrm{ns}$ & 0.2084 \\
Wild type (N2) vs. xpf-1-1(tm2842) & $* * *$ & $<0.0001$
\end{tabular}




\begin{tabular}{|lcc|}
$f c d-2(t m 1298)$ vs. $x p a-1(o k 698)$ & $n s$ & 0.8896 \\
$f c d-2(t m 1298)$ vs. $x p c-1(t m 3886)$ & $n s$ & 0.3876 \\
$f c d-2(t m 1298)$ vs. $x p f-1-1(t m 2842)$ & $* * *$ & $<0.0001$ \\
$x p a-1(o k 698)$ vs. $x p c-1(t m 3886)$ & $n s$ & 0.8638 \\
$x p a-1(o k 698)$ vs. $x p f-1-1(t m 2842)$ & $* * *$ & $<0.0001$ \\
$x p c-1(t m 3886)$ vs. $x p f-1-1(t m 2842)$ & $* * *$ & $<0.0001$ \\
\hline
\end{tabular}

Figure S3 (4 $\mu \mathrm{M}$ TMP)

\begin{tabular}{|lcc|}
\hline Tukey's multiple comparisons test & Significance & P Value \\
\hline Wild type (N2) vs. $f c d-2$ (tm 1298) & $*$ & 0.0348 \\
Wild type (N2) vs. $x p a-1(o k 698)$ & $* * *$ & $<0.0001$ \\
Wild type (N2) vs. $x p c-1(t m 3886)$ & $* * *$ & $<0.0001$ \\
Wild type (N2) vs. $x p f-1-1(t m 2842)$ & $* * *$ & $<0.0001$ \\
fcd-2(tm1298) vs. $x p a-1(o k 698)$ & $* * *$ & $<0.0001$ \\
fcd-2(tm1298) vs. $x p c-1(t m 3886)$ & $* * *$ & $<0.0001$ \\
fcd-2(tm1298) vs. $x p f-1-1(t m 2842)$ & $* * *$ & $<0.0001$ \\
$x p a-1(o k 698)$ vs. $x p c-1(t m 3886)$ & $n s$ & 0.9997 \\
$x p a-1(o k 698)$ vs. $x p f-1-1(t m 2842)$ & $n s$ & $>0.9999$ \\
$x p c-1(t m 3886)$ vs. $x p f-1-1(t m 2842)$ & $n s$ & 0.9993 \\
\hline
\end{tabular}

Figure S3 $(20 \mu \mathrm{M}$ TMP)

\begin{tabular}{|lcc|}
\hline Tukey's multiple comparisons test & Significance & P Value \\
\hline Wild type (N2) vs. $f c d-2$ (tm 1298) & $* * *$ & $<0.0001$ \\
Wild type (N2) vs. $x p a-1(0 k 698)$ & $* * *$ & $<0.0001$ \\
Wild type (N2) vs. $x p c-1(t m 3886)$ & $* * *$ & $<0.0001$ \\
Wild type (N2) vs. $x p f-1-1(t m 2842)$ & $* * *$ & $<0.0001$ \\
fcd-2(tm1298) vs. $x p a-1(o k 698)$ & $* * *$ & $<0.0001$ \\
$f c d-2(t m 1298)$ vs. $x p c-1(t m 3886)$ & $* * *$ & $<0.0001$ \\
$f c d-2(t m 1298)$ vs. $x p f-1-1(t m 2842)$ & $* * *$ & $<0.0001$ \\
$x p a-1(o k 698)$ vs. $x p c-1(t m 3886)$ & $n s$ & $>0.9999$ \\
$x p a-1(o k 698)$ vs. $x p f-1-1(t m 2842)$ & $n s$ & 0.9992 \\
$x p c-1(t m 3886)$ vs. $x p f-1-1(t m 2842)$ & $n s$ & 0.9997 \\
\hline
\end{tabular}

